\title{
MOSFET-C Filter with Low Excess Noise and Accurate Automatic Tuning
}

\author{
Jaap van der Plas
}

\begin{abstract}
Continuous-time integrated filters having a maximum dynamic range at the audio-frequency range are obtained using MOSFET-C filters. In combination with a careful design of the applied symmetrical amplifiers in a BiCMOS technology, the detrimental influence of $1 / f$ noise can be minimized. $A$ 98-dB dynamic range (THD $<0.3 \%$ ) is obtained in a realized fifth-order Butterworth low-pass filter, while the overall $1 / f$ noise corner frequency is limited to $250 \mathrm{~Hz}$. Automatic adjustment of the filter cutoff frequency is performed using a novel all-silicon automatic tuning system that, compared with traditional automatic tuning systems, demands reduced overhead circuitry.
\end{abstract}

\section{INTRODUCTION}

$\mathrm{T}$ HE achievement of a sufficiently high dynamic range in combination with a high linearity, a minimum chip area, and minimum power consumption is generally the main objective for continuous-time integrated filters. An important figure of merit that combines noise performance with linearity is the intermodulation-free dynamic range (IMFDR). The IMFDR is defined as the ratio between that level of two sine waves with equal amplitude for which the level of the intermodulation products is equal to the noise floor and the noise floor itself.

A major limitation of integrated technology is the $a b$ sence of usable inductors, while only small capacitors are available as reactive elements. Consequently, the requirement of a filter transfer with complex poles can only be met using active filters. A basic building block of continuous-time active filters is an active integrator. Maximization of the (intermodulation-free) dynamic range of the entire filter implies both optimization of a single integrator and optimization of the filter structure [1]. The implementation of active integrators having a maximum (intermodulation-free) dynamic range for audio frequencies for a given chip area and power consumption is dealt with in Section II. Subsequently, Section III describes the design of a symmetrical amplifier applied in MOSFET-C filters.

The transfer of a filter in the frequency domain can be normalized with respect to a certain cutoff frequency (or central frequency). In integrated active filters, the (dimensionless) coefficients of the normalized filter transfer can

Manuscript received November 26, 1990; revised March 11, 1991. This work was supported by the Dutch Technology Foundation (STW).

The author is with the Department of Electrical Engineering, Delft University of Technology, 2628 CD Delft, The Netherlands.

IEEE Log Number 9100045. be established as ratios of on-chip component values, which are generally well matched. The cutoff frequency, however, is determined by absolute component values. The large absolute tolerance generally makes tuning of this cutoff frequency necessary. Section IV presents a novel automatic tuning system that, compared with traditional tuning systems, requires a reduced amount of overhead circuitry.

As an application example, Section $\mathrm{V}$ presents a prototype continuous-time filter, implemented in a BiCMOS technology. The application of this filter as an output filter of a synchronous detector for an AM shortwave upconversion receiver [2], [3] requires a fifth-order Butterworth low-pass filter with a $3.3-\mathrm{kHz}$ cutoff frequency, having a dynamic range of at least $90 \mathrm{~dB}$ (THD $<0.3 \%$ ) and a second- and third-order IMFDR of at least 70 and $77 \mathrm{~dB}$, respectively. The applied calculation model for MOS transistors is outlined in the Appendix.

\section{INTEGRATOR IMPLEMENTATIONS}

An active integrator is realized around a resistive voltage-to-current transfer and a capacitor as a current-tovoltage integrator. In order to facilitate automatic tuning, the integration time constant has to be electrically adjustable. Since (fixed) oxide capacitors are largely preferred because of the excellent linearity and matching, this facility requires electrically adjustable resistive voltage-to-current transfers.

Electrically adjustable resistive transfers are commonly realized using MOS transistors as transconductance elements or as gate-controlled resistors. Balanced integrator structures are applied in order to cancel even-order nonlinearity emerging from the quadratic behavior of MOS transistors. For low-frequency applications $(<100 \mathrm{kHz})$, special attention must be paid to the detrimental influence of $1 / f$ noise (also called excess noise or flicker noise) upon the dynamic range.

Fig. 1 shows an elementary integrator architecture, built up around a source-coupled pair as a transconductance element. Using the square-law drain-current model in the saturation region, given in the Appendix, the small-signal integrator transfer is calculated as

$$
\frac{V_{o}(s)}{V_{i}(s)}=\frac{1}{s \tau}=\frac{\sqrt{\beta I_{T}}}{2 s C}=\frac{g_{m}}{2 s C}
$$




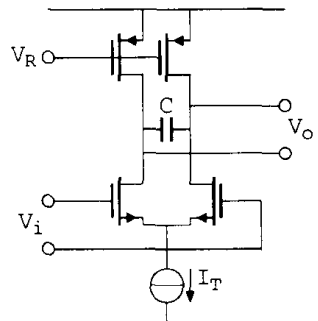

Fig. 1. Elementary integrator architecture.

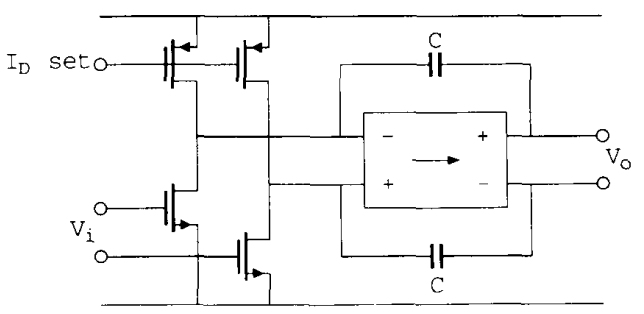

Fig. 2. Alternative transconductance integrator.

The small-signal transconductance $g_{m}$ and hence the integration time constant $\tau$ are adjusted by the tail current $I_{T}$.

From the Appendix (equation (A6)), it follows that the equivalent input noise voltage in a frequency range $\Delta f$ can be calculated as

$$
V_{n i}^{2}=\frac{8 k T \tau}{3 C} \int_{\Delta f}\left[1+\frac{f_{l n}}{f}+\frac{g_{m p}}{g_{m n}}\left(1+\frac{f_{l p}}{f}\right)\right] d f
$$

where the indexes $n$ and $p$ indicate the NMOS and PMOS transistor, respectively. The frequency $f_{l}$ expresses the noise corner frequency for which the $1 / f$ noise level equals the thermal noise level. For drain currents of about $10 \mu \mathrm{A}, f_{l n}$ is in the order of magnitude of $300 \mathrm{kHz}$ and $f_{l p}$ is in the order of magnitude of $10 \mathrm{kHz}$ in the applied process. Comparable results are obtained for a PMOS source-coupled pair using NMOS current sources.

An alternative transconductance integrator, depicted in Fig. 2, uses NMOST's biased in the triode region. According to the Appendix, a constant drain-source voltage results in a linear transfer from the gate-source voltage to the drain current. With $V_{D S}$ the drain-source bias voltage, the integrator transfer is equal to

$$
\frac{V_{o}(s)}{V_{i}(s)}=\frac{1}{s \tau}=\frac{\beta V_{D S}}{s C}=\frac{g_{m}}{s C} .
$$

This drain-source bias voltage and hence the integration time constant is adjusted by the PMOS current sources.

In analogy with the saturated transconductance integrator, the equivalent input noise voltage in a frequency

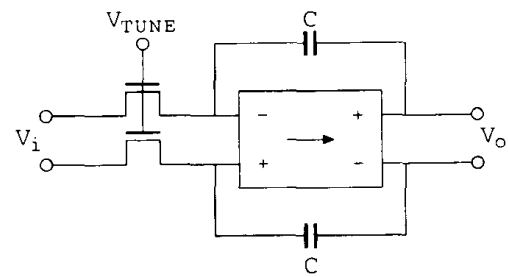

Fig. 3. Principle of MOSFET-C integrator using gate-controlled MOS resistors.

range $\Delta f$ is calculated as

$$
V_{n i}^{2}=\frac{8 k T \tau}{C} \int_{\Delta f}\left[\frac{V_{G}}{V_{D S}}\left(1+\frac{f_{l^{\prime} n}}{f}\right)+\frac{2 g_{m p}}{3 g_{m n}}\left(1+\frac{f_{l p}}{f}\right)\right] d f \text {. }
$$

The $1 / f$ noise corner frequency $f_{l}$ in the triode region is much smaller than the corner frequency in the saturation region. In the applied process, this corner frequency is in the order of magnitude of $50 \mathrm{kHz}$ for NMOST's using $I_{D} \approx 10 \mu \mathrm{A}$. If the amplifier is optimally designed, its noise contribution can be kept lower than that of the triode transconductance transistors.

Fig. 3 shows the principle of a MOSFET-C integrator that uses gate-controlled MOS resistors. According to the Appendix, the equivalent resistance of a gate-controlled MOS resistor with zero bias current is calculated as

$$
R_{m}=\left.\frac{d V_{D S}}{d I_{D}}\right|_{V_{D S}=0}=\frac{1}{\beta V_{G}}
$$

which results in the integrator transfer

$$
\frac{V_{o}(s)}{V_{i}(s)}=\frac{1}{s \tau}=\frac{1}{s R_{m} C} .
$$

The thermal noise of a MOS resistor with zero bias current corresponds to that of its ohmic equivalent, while the $1 / f$ noise is totally absent. Provided that the symmetrical amplifier is well designed, the equivalent input noise voltage of this integrator is completely determined by the thermal noise of the MOS resistors:

$$
V_{n i}^{2}=8 k T R_{m} \Delta f=\frac{8 k T \tau \Delta f}{C} .
$$

If we compare the equivalent input noise voltages of the given integrator implementations for equal values of the time constant $\tau$ and the capacitance $C$, the MOSFET-C integrator has the lowest noise level, especially for low frequencies. The signal handling capability of these integrators does not significantly differ, since they are bounded by the available supply voltage. Consequently, MOSFET-C filters provide the highest dynamic range for audio-frequency applications.

Calculation of the distortion of MOSFET-C filters is based upon the drain current model given in the Appendix. From (A4), the differential-mode input current as a function of the differential-mode input voltage of the 
MOSFET-C integrator is derived as

$$
i_{d \mathrm{l}}-i_{d 2}=\beta\left\{V_{G} v_{i}-\frac{\epsilon}{2} v_{i}^{2}+\frac{\gamma}{96} V_{B}^{-3 / 2} v_{i}^{3}\right\}
$$

In this equation, $\epsilon$ denotes a small imbalance in transistor parameters. Measurements of the applied MOS resistors show that the effect of mobility degradation can practically be ignored in this specific case. As a result, the second- and third-order IMFDR's are calculated as

$$
\begin{aligned}
\text { IMFDR }_{2} & =\sqrt[4]{\frac{V_{G}^{2} C}{4 \epsilon^{2} k T \tau \Delta f}} \\
\text { IMFDR }_{3} & =\sqrt[3]{\frac{8 V_{B}^{3 / 2} V_{G} C}{\gamma k T \tau \Delta f}} .
\end{aligned}
$$

The maximum input voltage for which the MOS transistors remain in the triode region is almost equal to $2 V_{G}$, which results in a dynamic range:

$$
\mathrm{DR}=\sqrt{\frac{V_{G}^{2} C}{4 k T \tau \Delta f}} .
$$

Additional odd-order nonlinearity cancellation by the double-MOSFET method is not applied, since this method increases the thermal noise level and does not compensate for the residual even-order nonlinearity due to component mismatches [5].

\section{Design of a Symmetrical Amplifier}

A MOSFET-C integrator requires a fully balanced amplifier with a sufficiently high differential-mode voltage gain and an accurate output common-mode voltage. The limited value of on-chip capacitances combined with a low cutoff frequency requires large MOS resistors $R_{m}$ in the order of magnitude of $100 \mathrm{k} \Omega$ to $1 \mathrm{M} \Omega$. The equivalent input noise voltage of the amplifier must not exceed the noise voltage of these resistors.

Optimum noise matching with the MOS resistors in combination with a maximum gain and a high commonmode rejection is obtained using a PMOS source-coupled pair as input stage. The transfer and noise behavior of a similar source-coupled pair was already dealt with in Section II. As mentioned before, the $1 / f$ noise corner frequency of PMOS transistors is much lower than that of NMOS transistors. The common-mode rejection is important in order to prevent common-mode instability by parasitic common-mode loops within the filter.

The current sinks of the input stage are commonly implemented using NMOS transistors. At low frequencies, the $1 / f$ noise of these NMOS transistors dominates over the noise of the PMOS input stage and even over the thermal noise of the MOS resistors. If these current sinks are replaced by ordinary resistors, the resulting loss of amplifier gain is generally unacceptable, unless a bipolar second stage is applied. Current sinks using bipolar transistors are not considered as a useful alternative because of the high level of the collector-current shot noise.

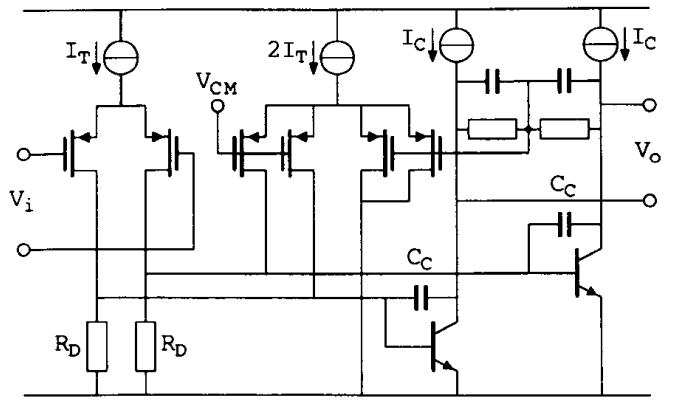

Fig. 4. Two-stage BiCMOS amplifier with common-mode feedback.

A two-stage BiCMOS amplifier, using a bipolar common-emitter stage as second stage, is depicted in Fig. 4. The output voltage swing of this bipolar output stage is nearly rail to rail. Accurate common-mode sensing is performed using two diffused resistors and the double source-coupled pair provides for a maximum commonmode accuracy [3], [4]. The capacitors $C_{C}$ are added for phase compensation.

The small-signal low-frequency differential-mode voltage gain of the amplifier according to Fig. 4 is calculated as

$$
G_{d m}=g_{m p} \frac{R_{D}}{R_{D}+r_{\pi}} \beta_{f} R_{L}
$$

where $g_{m p}=\sqrt{\beta_{p} I_{T}}$ is the transconductance of the PMOS input transistor and $\beta_{f}$ is the current gain of the n-p-n transistors. The common-mode sense resistors are part of the effective load resistance $R_{L}$, calculated from each output to ground. The input impedance $r_{\pi}$ of the bipolar output stage is equal to

$$
r_{\pi}=\frac{\beta_{f} V_{T}}{I_{C}}
$$

in which $V_{T}$ is the thermal voltage. With $R_{D}=V_{B E} / I_{T}$, (12) can be written as

$$
G_{d m}=\sqrt{\beta_{p} I_{T}} \frac{V_{B E}}{V_{B E}+\beta_{f} V_{T} I_{T} / I_{C}} \beta_{f} R_{L} .
$$

If the base current is ignored, a maximum voltage gain for a given collector current $I_{C}$ is found if

$$
I_{T}=\frac{V_{B E} I_{C}}{\beta_{f} V_{T}}
$$

and consequently, $R_{D}=r_{\pi}$. This maximum voltage gain is equal to

$$
G_{d m}=\frac{1}{2} g_{m p} \beta_{f} R_{L}
$$

The equivalent input noise voltage of the amplifier for $R_{D} \approx r_{\pi}$ is completely determined by that of the input 


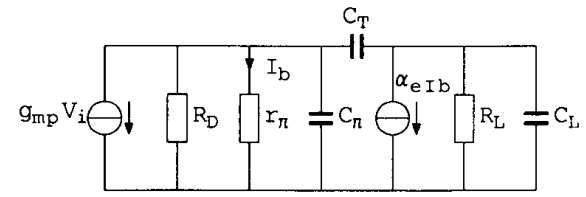

Fig. 5. Single-ended amplifier model for calculation of high-frequency behavior.

source-coupled pair:

$$
S\left(v_{n i}\right)=\frac{16 k T}{3 g_{m p}}\left(1+\frac{f_{l p}}{f}\right) .
$$

With a low bias current $I_{T}$ of some tens of microamperes, the $1 / f$ noise corner frequency $f_{l p}$ of the PMOS transistors is limited to $10 \mathrm{kHz}$ or even lower. The $1 / f$ noise can be neglected in the weighed audio spectrum if the overall corner frequency of the integrator does not exceed $500 \mathrm{~Hz}$, which results in the condition

$$
g_{m p}>\frac{40}{3 R_{m}} \text {. }
$$

In this case, the filter noise is determined by the noise of the integration resistors only.

For high frequencies $(f \gg 1 / \tau)$, the integration capacitors can be regarded as a unity feedback. A single-ended amplifier model for the calculation of the high-frequency behavior is depicted in Fig. 5. In this figure, $C_{\pi}$ is the bipolar transistor base-emitter capacitance, while the collector-base capacitance is incorporated in the compensation capacitance $C_{T}$. The input capacitance of the PMOS input stage and the parasitic substrate capacitance of the filter capacitors are part of the total load capacitance $C_{L}$. The drain resistor $R_{D}$ is assumed to be equal to $r_{\pi}$.

If we assume $\beta_{f} \gg 1$ and $\beta_{f} R_{L} \gg r_{\pi}$, the poles in the amplifier transfer can be calculated as

$$
\begin{aligned}
& p_{1} \approx-\frac{1}{R_{L}\left(C_{L}+\beta_{f} C_{T} / 2\right)} \\
& p_{2} \approx-\frac{2+\beta_{f} C_{T} / C_{L}}{r_{\pi}\left(C_{T}+C_{\pi}\right)}
\end{aligned}
$$

in which $p_{1}$ is the most dominant one $\left(p_{1} \ll p_{2}\right)$. The resulting unity-gain angular frequency $\omega_{t}$ is equal to

$$
\omega_{t}=-G_{d m} p_{1}=\frac{g_{m p}}{C_{T}+2 C_{L} / \beta_{f}} .
$$

Since the most dominant pole $p_{1}$ gives a phase lag of $90^{\circ}$ at $\omega_{t}$, the phase margin $\phi_{m}$ is calculated as

$$
\phi_{m}=90^{\circ}-\arctan \left(\frac{G_{d m} p_{1}}{p_{2}}\right)
$$

in which

$$
\frac{G_{d m} p_{1}}{p_{2}}=\frac{g_{m p}}{g_{m n}} \frac{\left(C_{T}+C_{\pi}\right) C_{L}}{\left(C_{T}+2 C_{L} / \beta_{f}\right)^{2}}
$$

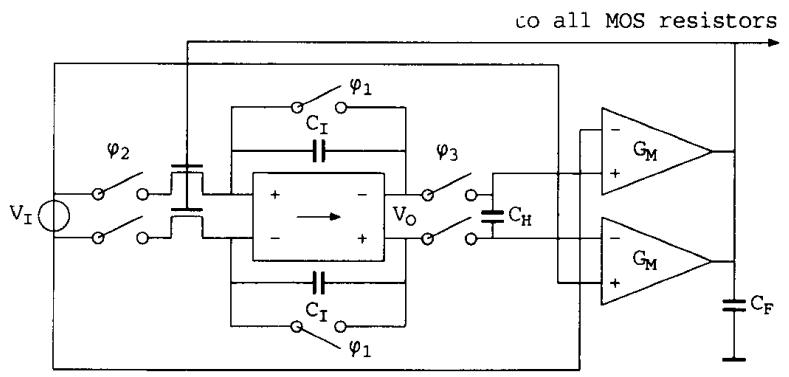

Fig. 6. Novel elegant tuning technique.

In this equation, $g_{m n}=I_{C} / V_{T}$ is the transconductance of the bipolar output transistors. A minimum required phase margin of $45^{\circ}$ results in the relation

$$
\frac{g_{m n}}{g_{m p}} \geqslant \frac{\left(C_{T}+C_{\pi}\right) C_{L}}{\left(C_{T}+2 C_{L} / \beta_{f}\right)^{2}} .
$$

The small-signal behavior of the common-mode feedback loop is similar to the unity-feedback differentialmode behavior. The common-mode loop gain, however, is approximately half the differential-mode gain $G_{d m}$. Since the phase lag of the common-mode sense resistors is compensated by two bypass capacitors (see Fig. 4), differential-mode stability also implies common-mode stability.

In an implementation of a fifth-order MOSFET-C filter, described in Section $\mathrm{V}$, the symmetrical amplifier is dimensioned using a $0.1-\mathrm{mA}$ collector current for the bipolar output transistors. With $V_{B E}=0.7 \mathrm{~V}, \beta_{f} \approx 190$ $\mu \mathrm{A} / \mathrm{V}^{2}$, and $\beta_{p}=250 \mu \mathrm{A} / \mathrm{V}^{2}$, a maximum voltage gain is obtained if $R_{D}=r_{\pi}=48 \mathrm{k} \Omega$ and $I_{T}=15 \mu \mathrm{A}$. The transconductance of the input transistor $g_{m p}=61 \mu \mathrm{A} / \mathrm{V}$. The maximum voltage gain is $49 \mathrm{~dB}$ for an effective load resistance $R_{L}=50 \mathrm{k} \Omega$. Using a $1-\mathrm{pF}$ compensation capacitance, the calculated unity-gain bandwidth is $6.4 \mathrm{MHz}$ with a $57^{\circ}$ phase margin. The output capacitance in this case is $50 \mathrm{pF}$ for each output to ground. The measured transfer does not significantly differ from the calculated one $\left(G_{d m}=52 \mathrm{~dB}, \mathrm{GBW}=6 \mathrm{MHz}\right.$, and $\left.\phi_{m}=55^{\circ}\right)$.

\section{Automatic Tuning}

In continuous-time integrated filters, tuning of the cutoff frequency is usually performed automatically by means of indirect tuning techniques. The integration time constants of one or more additional integrators, implemented on the same chip as the filter itself, are related to a reference frequency. If all integrators are simultaneously tuned, the value of the cutoff frequency is related to the reference frequency as well. The accuracy of such tuning techniques is determined by the matching of on-chip component values.

Traditional indirect automatic tuning concepts use two active integrators configured as a two-integrator oscillator in a PLL or as a frequency discriminator in a frequency control loop [5]. Fig. 6 shows a novel elegant tuning technique, in which the integration time constant of only 


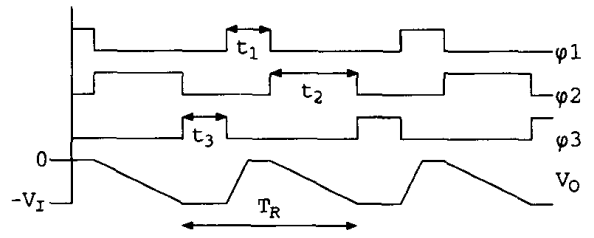

Fig. 7. Timing diagram.

one additional integrator is compared with a time reference. The timing and control is performed directly with switches, which are driven by the reference timing signals $\varphi_{1}, \varphi_{2}$, and $\varphi_{3}$. This technique is called the direct sample tuning technique.

The timing diagram is shown in Fig. 7. During the time $\varphi_{1}$ is active, the integration capacitors are short circuited, forcing the output differential-mode voltage to zero. During the time interval $t_{2}$, in which $\varphi_{2}$ is active, a fixed differential input voltage is connected to the integrator input, which results in a ramp voltage at the integrator output. Finally, when $\varphi_{3}$ gets active, the integrated voltage is sampled into the capacitor $C_{H}$. This sampled voltage passes through the low-pass filter, consisting of two transconductances $G_{M}$ and a capacitor $C_{F}$, to the gates of all MOS resistors.

In the stationary state, the final sampled voltage $V_{O}$ is equal to the reference voltage $V_{I}$, which is also the integrator input voltage. Consequently, the integration time constant $\tau_{I}$ is tuned to the time interval $t_{2}$, which is derived from a reference frequency. The sampled and filtered tuning voltage, applied to all MOS resistors, remains constant with no ripple. Now all absolute values of all time constants, and hence the cutoff frequency, are related to the reference time $t_{2}$.

The stability of the sample control loop would pose no problems if the time constant $C_{F} / G_{M}$ is made much larger than the autotuning cycle time $T_{R}$. However, if all capacitors including $C_{F}$ have to be implemented on chip, only a limited time constant $C_{F} / G_{M}$ can be realized. Because the autotuning circuitry inherently produces no ripple on the resistor tuning voltage in the stationary state, the minimum $C_{F} / G_{M}$ mainly depends on the loop stability. Using the $z$ transform [3], the poles of the sample control loop are found with the root-locus equation:

$$
\frac{-1}{k}=\frac{3 z+1}{8 z^{2}-8 z} .
$$

The time interval $t_{2}$ is assumed to be half the tuning cycle time $T_{R}$, while the root-locus factor $k$ is equal to

$$
k=\frac{2 \beta V_{I} G_{M} T_{R}^{2}}{C_{I} C_{F}} .
$$

In this equation $\beta$ is the proportionality factor of the MOS resistors. The root locus of this sampled feedback system as a function of $k$ is drawn in Fig. 8 .

The breakaway point in the root locus at the real axis occurs for $k=8 / 9$ at $z=1 / 3$ and for $k=8$ at $z=-1$.

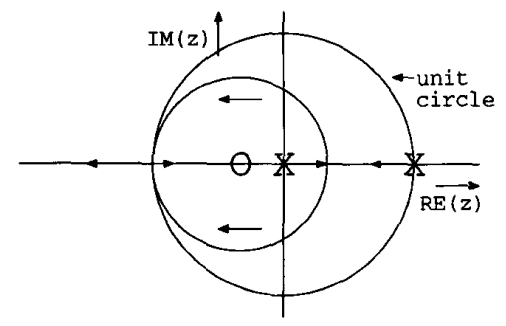

Fig. 8. Root locus of sampled feedback system as a function of $k$.

For a stable autotuning system without complex poles, we have to choose $k \leqslant 8 / 9$. However, a smaller value of $k$ is preferred for minimum noise and interference on the tuning voltage. In a prototype integrated filter, described in Section $\mathrm{V}, \beta=1 \mu \mathrm{A} / \mathrm{V}^{2}, V_{I}=1 \mathrm{~V}, T_{R}=32 \mu \mathrm{s}$, and $C_{I}=33 \mathrm{pF}$. A value of $k=0.4$ is obtained taking $G_{M}=$ $1.25 \mu \mathrm{A} / \mathrm{V}$ and $C_{F}=200 \mathrm{pF}$. Both $C_{I}$ and $C_{F}$ are implemented on-chip.

\section{Application Example}

This section presents a prototype fifth-order Butterworth filter, designed as an output filter of a synchronous detector for an AM shortwave upconversion receiver. For $\mathrm{HF}$ interference suppression purposes, this filter has to be implemented as a fourth-order active filter preceded by a first-order passive $R C$ filter [2], [3]. Fig. 9 shows a photomicrograph of the evaluation chip, on which the fourth-order MOSFET-C filter part (Fig. 11) including a first-order MOSFET-C test section (Fig. 10) and the automatic tuning circuitry (see Section IV) have been integrated.

A maximum signal-handling capability for signals within the filter passband requires a unity transfer from the filter input to the output of the integrators for frequencies below the cutoff frequency. For this reason, all integration resistors of one integrator section have the same value.

In order to obtain a Butterworth transfer:

$$
|H(s)|^{2}=\frac{\left|V_{o}\right|^{2}}{\left|V_{i}\right|^{2}}=\frac{1}{1+\left(\omega / \omega_{c}\right)^{10}}
$$

the integration time constants have to fulfill the relations:

$$
\begin{aligned}
& a_{1}=\omega_{c} \tau_{1}=1 \\
& a_{2}=\omega_{c} \tau_{2}=0.5 \sqrt{5} \\
& a_{3}=\omega_{c} \tau_{3}=0.8 \sqrt{5} \\
& a_{4}=\omega_{c} \tau_{4}=0.5 \sqrt{5} \\
& a_{5}=\omega_{c} \tau_{5}=0.2 \sqrt{5} .
\end{aligned}
$$

The angular cutoff frequency is equal to

$$
\omega_{c}=\frac{1}{\sqrt[5]{\tau_{1} \tau_{2} \tau_{3} \tau_{4} \tau_{5}}} .
$$

The dimensionless normalized filter coefficients $a_{i}$ are in 


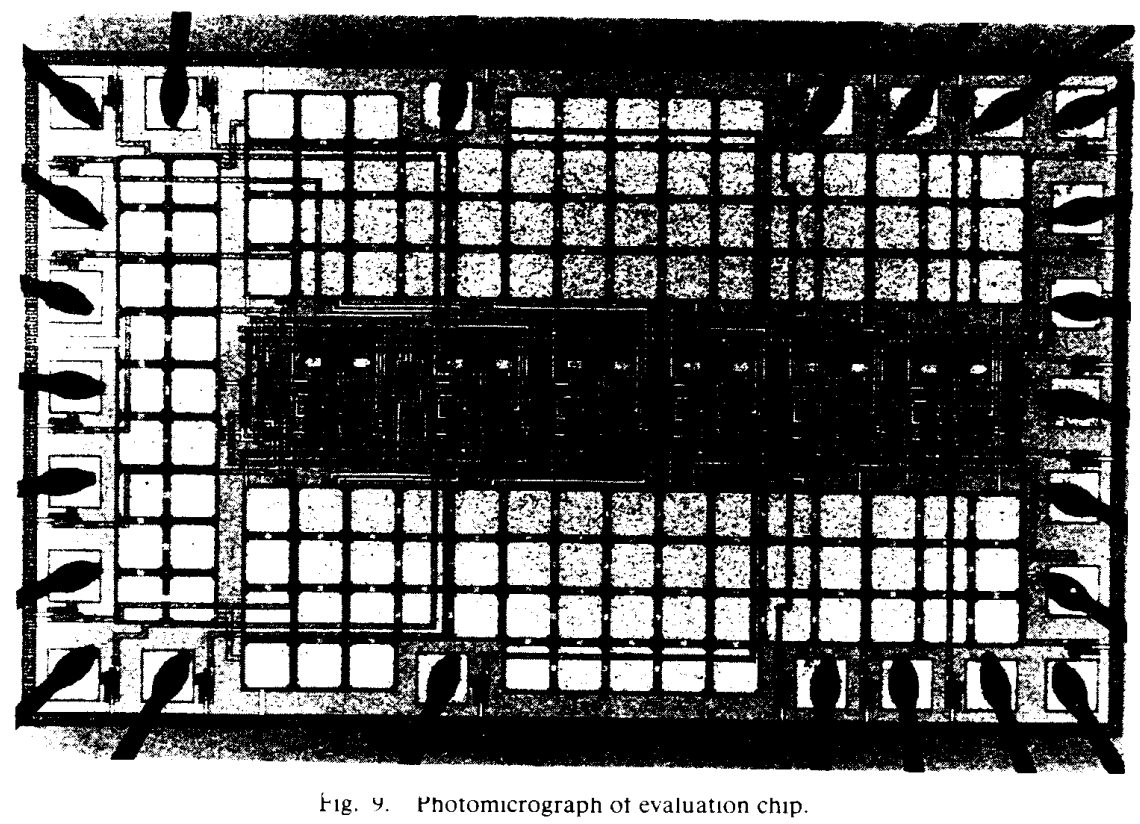

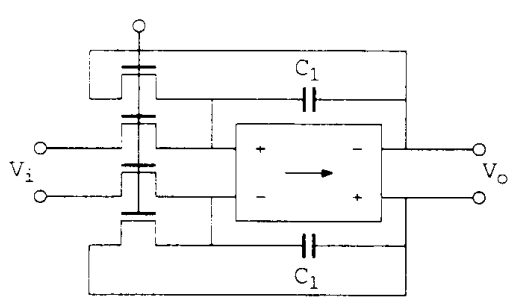

Fig. 10. First-order MOSFET-C test section.

fact ratios of component values. If all time constants are tuned with the same scale factor, the cutoff frequency can be adjusted without affecting the normalized coefficients and hence shape of the filter transfer. For MOSFET-C filters, this factor depends on the gate control voltage of the MOS resistors (see Section II), which is generated by the automatic tuning system.

The equivalent output noise voltage of the first-order section is calculated as

$$
V_{n \circ 1}^{2}=\frac{16 k T R_{m 1}}{2 \pi} \int_{0}^{\infty} \frac{d \omega}{1+\omega^{2} \tau_{1}^{2}}=\frac{4 k T}{C_{1}}
$$

$$
\text { i }
$$

which is, independent of the integration resistance, inversely proportional with the available capacitance. In analogy with this first-order section, the equivalent output noise of the fourth-order section is calculated as

$$
V_{\text {no4 }}^{2}=4 k T \sum_{i=2}^{5} \frac{1}{C_{i}}
$$

and the equivalent output noise voltage of the complete

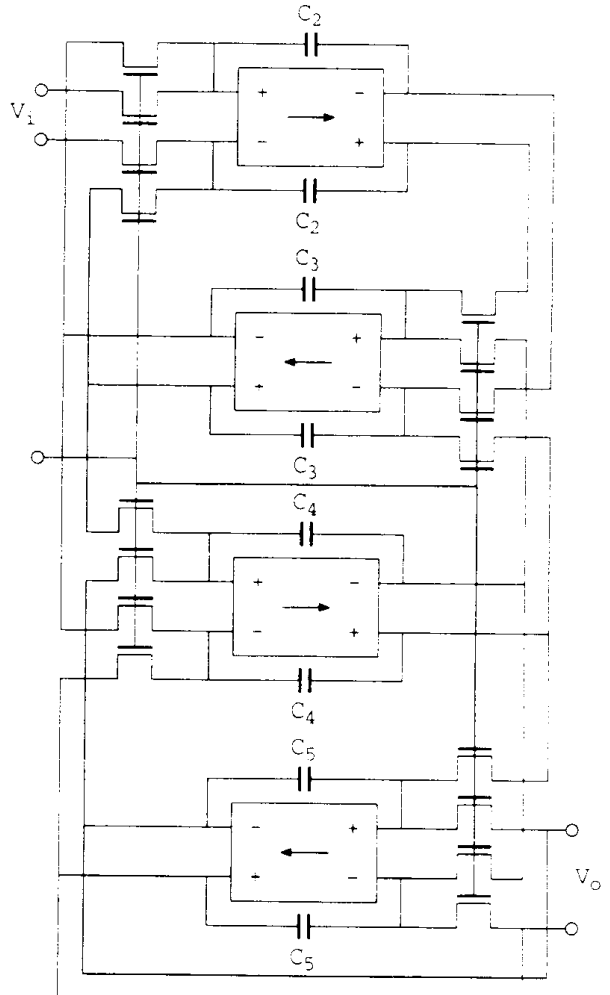

Fig. 11. Fourth-order MOSFET-C filter part.

filter is equal to

$$
V_{n o}^{2}=4 k T \sum_{i=1}^{5} \frac{1}{C_{i}} \text {. }
$$


If all MOS resistors are equal and the ratios of the time constants are established as ratios of the capacitor values, the output noise as a function of the total capacitance $\mathcal{C}_{T}$ is expressed by

$$
V_{n o}^{2}=\frac{8 k T}{C_{T}} \sum_{i=1}^{5} \frac{1}{a_{i}} \sum_{j=1}^{5} a_{j} \approx \frac{244 k T}{C_{T}} .
$$

However, if all capacitor values are equal and the ratios of the time constants are established as ratios of the MOS resistor values of each integrator section, the output noise as a function of the total capacitance $C_{T}$ is expressed by

$$
V_{n o}^{2}=\frac{200 k T}{C_{T}}
$$

which is only $1 \mathrm{~dB}$ lower. For a maximum tracking accuracy of the MOS resistors, all MOS resistors are taken as equal and a 1-dB higher noise floor is accepted.

In a silicon prototype, all MOS resistors $R_{m}$ have the nominal value of $500 \mathrm{k} \Omega$, which results in the capacitor values:

$$
\begin{aligned}
& C_{1}=99 \mathrm{pF} \\
& C_{2}=110 \mathrm{pF} \\
& C_{3}=176 \mathrm{pF} \\
& C_{4}=110 \mathrm{pF} \\
& D_{5}=44 \mathrm{pF} .
\end{aligned}
$$

With a total capacitance $C_{T}=1.078 \mathrm{nF}$, the calculated equivalent output noise voltage is $31 \mu \mathrm{V}$. Within the filter passband, the calculated noise density of the output voltage corresponds to that of a $10-\mathrm{M} \Omega$ resistor $\left(S\left(V_{n o}^{2}\right)=80\right.$ $\left.k T R_{m}\right)$.

Fig. 12 shows the measured filter transfers from the filter input to the integrator outputs. Within the passband of the filter, all integrators have to handle the same signal level. With a maximum 2-dB overshoot around the cutoff frequency, the signal-handling capability of the complete filter is almost equal to that of a single integrator. The measured filter output noise corresponds to the calculated output noise within $1 \mathrm{~dB}$, while the measured overall $1 / f$ noise corner frequency does not exceed $250 \mathrm{~Hz}$.

Using a nominal gate voltage $V_{G}=2.2 \mathrm{~V}$, the measured maximum input signal level for which the total harmonic distortion of one sine wave does not exceed $0.3 \%$ is $3.5 \mathrm{~V}_{\mathrm{p}}$ $(2.5 \mathrm{~V} \mathrm{rms})$. In combination with an output noise voltage $V_{n o}=31 \mu \mathrm{V}$, the dynamic range is $98 \mathrm{~dB}$. If we consider two interfering signals in the stopband, the intermodulation distortion is determined by the first MOS resistor pair. In this case, the IMFDR is calculated as

$$
\begin{gathered}
\operatorname{IMFDR}_{2}=\sqrt[4]{\frac{2 V_{G}^{2}}{\epsilon^{2} V_{n o}^{2}}} \\
\mathrm{IMFDR}_{3}=\sqrt[3]{\frac{64 V_{B}^{3 / 2} V_{G}}{\gamma V_{n o}^{2}}} .
\end{gathered}
$$

With $\epsilon=1 \%, V_{B}=3.5 \mathrm{~V}$, and $\gamma=0.28$, the calculated

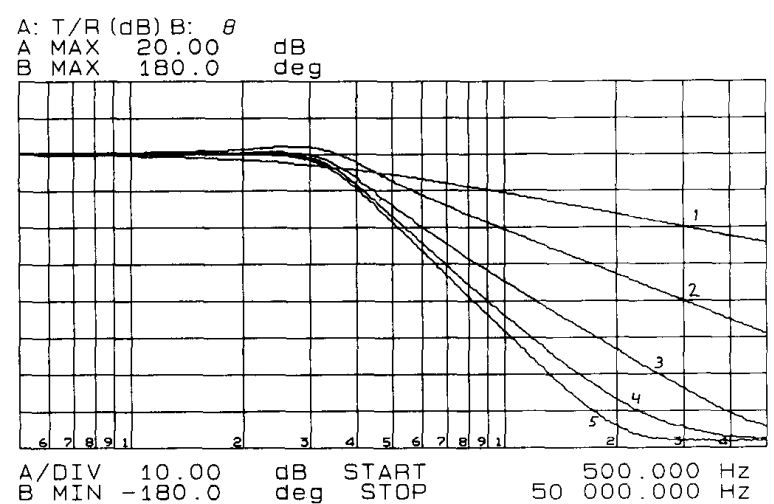

Fig. 12. Measured filter transfers from filter input to integrator outputs.

second- and third-order IMFDR's are 70 and $83 \mathrm{~dB}$, respectively. The measured second- and third-order IMFDR's are 73 and $82 \mathrm{~dB}$, respectively, which is very close to the calculated ones.

\section{Conclusions}

In conclusion, we can state that for continuous-time integrated filters in the audio-frequency range, MOSFET$\mathrm{C}$ filters are preferred over transconductance implementations. Using MOSFET-C filters in combination with a well-designed BiCMOS amplifier, the detrimental influence of $1 / f$ noise can practically be eliminated and the filter noise is completely determined by the unavoidable thermal noise of the filter resistors.

The novel direct sample tuning system proposed in Section IV provides for an all-silicon tuning system with minimum overhead circuitry.

\section{APPENDIX \\ APPLIEd MOS Model}

The applied MOS drain current formula in the triode region is derived from the Ihantola and Moll model, which can be found in $[6$, p. 120]:

$$
\begin{aligned}
I_{D} & =\beta\left(\left(V_{G S}-V_{F B}-\phi_{B}\right) V_{D S}-\frac{1}{2} V_{D S}^{2}\right. \\
& \left.+\frac{2}{3} \gamma\left(V_{S B}+\phi_{B}\right)^{3 / 2}-\frac{2}{3} \gamma\left(V_{S B}+V_{D S}+\phi_{B}\right)^{3 / 2}\right)
\end{aligned}
$$

In this equation, $V_{S_{B}}$ denotes the source-bulk voltage, $V_{F B}$ denotes the flat-band voltage, $\phi_{B}$ denotes the surface Fermi potential, and $\gamma$ denotes the body coefficient.

A simplified expression with a minimum loss of accuracy can be obtained using a Taylor-series expansion up to the third order:

$$
\begin{aligned}
I_{D}= & \beta\left(\left(V_{G S}-V_{T H}\right) V_{D S}-\left(\frac{1}{2}+\frac{\gamma}{4} V_{B}^{-1 / 2}\right) V_{D S}^{2}\right. \\
& \left.+\frac{\gamma}{24} V_{B}^{-3 / 2} V_{D S}^{3}\right) .
\end{aligned}
$$


In this equation, $V_{S B}+\phi_{B}$ is written as $V_{l}$ for short, while the threshold voltage $V_{T H}$ is equal to

$$
V_{T H}=V_{I B}+\phi_{B}+\gamma \sqrt{V_{B}} .
$$

For (Bi)CMOS processes with lightly doped substrates, the body factor $\gamma$ is small $(0.3 \sqrt{V})$ and the drain current formula can be further simplified to

$$
I_{D}=\beta\left(V_{G} V_{D S}-\frac{1}{2} V_{D S}^{2}+\frac{\gamma}{24} V_{B}^{-3 / 2} V_{D S S}^{3}\right) .
$$

The term $V_{G s}-V_{T H}$ is written as $V_{G}$ for short. This equation is valid for $V_{G}>V_{D, S}$. In the saturation region, for which $V_{G}<V_{D S}$, the drain current is modeled according to the square law:

$$
I_{D}=\frac{\beta}{2} V_{(i}^{2}
$$

The drain-current spectral noise density of a MOS transistor is given by

$$
S\left(I_{d n}\right)=4 k T \beta V_{c_{i}} \frac{2}{3} \frac{1+\alpha+\alpha^{2}}{1+\alpha}+\frac{\left.K_{F} I_{i}\right)^{\prime}}{W L C_{i x}} \frac{1}{f} .
$$

The left-hand term expresses the thermal noise according to [6], where $k$ is the Boltzman constant and $T$ is the absolute temperature. In the triode region, the factor $\alpha$ is equal to:

$$
\alpha=1-\frac{V_{D S}}{V_{G}}
$$

while in the saturation region, $\alpha=0$. The right-hand part of (A6) expresses the $1 / f$ noise according to [7], where $K_{F}$ and $A_{F}$ are technology-dependent constants, $W$ is the transistor length, $L$ is the transistor width, and $C_{o x}$ is the oxide capacitance per unit area.

\section{A(KNOWLIET(IMENI}

Silicon prototypes of the filters were processed by Philips Nijmegen. The Netherlands.

\section{RFILRINCES}

[1] (i. Groenewold. "Design of high dynamic range continuous-time integrable bandpass filters." to be published in IEEE Trans. Circuits sist. vol. 38.1991

[2] J. van der Plas and E. H. Nordholt, "A novel extended dynamic range synchronous detector for AM shortwave upconversion receiver." IEEE Trans. Consumer Electron.. vol. 35. no. 3, pp. 390-396, Aug. 1989.

[3] J. van der Plas. "Synchronous detection in monolithically integrated AM upconversion receivers." Ph.D. dissertation. Delft Univ. of Technology, Delft. The Netherlands, Nov. 1990.

[4] M. Banu, J. M Khoury and Y Tsividis "Fully differential operational amplifiers with accurate output balancing, "ILEEJ. Solid-State (ircuits, vol. 23 , no. 6. pp. $1+10-1+17$. Dec. 1988.

[5] Y. Tsividis. B. Banu. and J. Khoury. "Continuous-time MOSFET-C fiiters in VLSI." HEEE Trans. Circuits Syst.. vol. CAS-33, no. 2. pp. $125-139$. Feh. 1986

[6] Y. P. Tsividis, Operation and Modeling of the MOS Transistor. New York: McGraw-Hill. 1987

[7] A. Vladimirescu and S. Liu. "The simulation of MOS integrated circuits using Spice2." College of Engineering. Univ. of Calit.. Berkeley. Memo. UCB/FRL M⿻日/7, Feb. 1980.

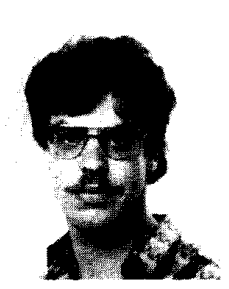

Jaap van der Plas was born in Leiden. The Netherlands, on April 3. 1963. He received the master's degree in electrical engineering from Delf University of Technology. Delft. The Netherlands in 1986. Subsequently, he joined the Flectronics Reseatch Laboratory at this uniwersity ats a Research Assistant. and received the Ph.D. degree on November 26, 1990. His Ph.D. research involved the design of integrated AM reccivers with synchronous detection.

Currently he is working at the Delft Institute of Microelectronics and Submicron Technology (DIMES). 